\title{
C-kit Expression in Oral Leukoplakia
}

\author{
Anand S. Tegginamani ${ }^{1}$, Vanishree H. Shivakumar ${ }^{1}$, Siti Mazlipah B. Ismail ${ }^{2}$, Mannil T. Abraham ${ }^{3}$, Bennete A. \\ Fernandes ${ }^{1}$ and Ahmad Termizi B. Zamzuri ${ }^{1}$ \\ ${ }^{1}$ Faculty of Dentistry, SEGi University, Kota Damansara, Petaling Jaya, Selangor, Malaysia \\ ${ }^{2}$ Faculty of Dentistry, University of Malaya, Kuala Lumpur, Malaysia \\ ${ }^{3}$ Department of Dentistry, Sentosa Specialist Hospital, Taman Sentosa Perdana, Klang, Selangor, Malaysia
}

\begin{abstract}
Oral leukoplakia is the most common potentially malignant oral disorder. Oral leukoplakia's malignant potential is independent of the histopathological grade, and the malignant transformation rate varies greatly from $3 \%$ to $50 \%$ even in the case of severe epithelial dysplasia. Ethnic \& environmental variables may contribute to this variation. C-kit immunohistochemistry was performed on 15 oral leukoplakia (OL), two oral squamous cell carcinoma (OSCC), and two dentigerous cysts (DC). The objective of this study was to evaluate the c-kit expression in oral leukoplakia. The use of various immunohistochemical markers to differentiate between OLs with a high and low risk of malignant transformation has been investigated. Only four OL exhibited a faint cytoplasmic expression in basal cells. Whereas, OSCC and DC were devoid of c-kit expression. Thus, this may not be a unique marker for identifying OL at high-risk. Further research with larger sample size is required.
\end{abstract}

Key Words: CD 117, Disease progression, Oral dysplasia, Oral leukoplakia, Risk prediction.

How to cite this article: Tegginamani AS, Shivakumar VH, Ismail SMB, Abraham MT, Fernandes BA, Zamzuri ATB. C-kit Expression in Oral Leukoplakia. J Coll Physicians Surg Pak 2022; 32(02):256-258.

Oral Leukoplakia $(\mathrm{OL})$ is the most common oral potentially malignant disorder (OPMD) or potentially premalignant oral epithelial lesion (PPOEL), which is associated with an increased risk of cancer. ${ }^{1}$ However, the risk varies with gender, location, type of lesion and habits. The presence of epithelial dysplasia is related to an increased risk of malignant transformation. ${ }^{1}$ Although it is generally understood that not all dysplastic lesions develop malignancy, ${ }^{1}$ even non-dysplastic lesion can lead to malignant transformation. Currently, it is difficult to predict an individual's malignant transformation using clinical and/or histological data. A biomarker that can discriminate between lesions that will develop into cancer and those that will not, is critically needed. The c-kit (CD117) proto-oncogene receptor tyrosine kinase is encoded by the c-kit proto-oncogene and is found in hematopoietic stem cells, melanocytes, mast cells, germ cells, and intestinal cells of Cajal. ${ }^{2}$ Its expression has also been detected in several solid tumors. ${ }^{1,2}$

Retrospective formalin-fixed paraffin-embedded tissue samples were collected. The tissue sample of oral epithelial dysplasia (OED)/oral leukoplakia tissues $(n=15)$, oral squamous cell carcinoma (OSCC) tissues ( $n=2)$, dentigerous cyst (DC) tissues $(n=2)$ were obtained from the Oral Cancer Research and Coordinating Centre, Malaysian Oral Cancer Database and Tissue Bank System, University of Malaya, Malaysia.

Correspondence to: Dr. Anand Tegginamani, Faculty of Dentistry, SEGi University, Kota Damansara, Petaling Jaya, Selangor, Malaysia

E-mail: anandsiddappa@segi.edu.my

Received: September 09, 2021; Revised: October 25, 2021; Accepted: November 09, 2021

DOI: https://doi.org/10.29271/jcpsp.2022.02.256
The current study evaluated thec- kit utilising DC samples of odontogenic origin. The study was approved by the Institutional Ethics Committee (Reference No. SEGilRF/2016-25/FOD-10/100).

After deparaffinisation and rehydration processes, immunohistochemistry was conducted on formalin-fixed and paraffinembedded $5 \mu \mathrm{m}$ thick slices of tissue samples, with epitope retrieval performed in hot citrate buffer. The primary antibody CD117 (DAKO Corporation, USA, 1; 500 polyclonal) immunohistochemical staining was done according to the manufacturer's instructions. ${ }^{2}$ Counter stained with Mayer's haematoxylin, the slides were cover slipped with DPX. The immunostained sections were examined under a light microscope to determine where the immunostaining was located within the epithelial tissue component. Positive controls included gastrointestinal stromal tumour (GIST), SCC of the cervix, and adenoid cystic carcinoma (ACC); whereas, negative controls included omitting the primary antibody. The percentage of $\mathrm{CD} 117$ expression was divided into four categories: ${ }^{2}$ Grade $0(0-19 \%)$, Grade 1 (20-39\%), Grade 2 (40-59\%), and Grade 3 (60-100\%). The staining intensity was used to grade the intensity of expression: weak, moderate, and strong. Immunohistochemistry was performed blindfolded; that is, without knowing the epithelial dysplasiahistological grade.

The c-kit was overexpressed Grade 3 with high intensity of staining in GIST $(n=1)$ (Figure 1a), moderate staining Grade 3 with ACC $(n=1)$ (Figure $1 b)$, moderate staining Grade 2 for SCC of the cervix (Figure 1c), and DC (2/2) were negative (Figure 1d). OSCC had no expression (2/2) (Figure 2a). Only 4/15 (26.6\%) of OED had Grade 0 cytoplasmic expression in basal cells (Figure $2 d)$ with weak intensity of staining, while $73.4 \%$ (11/15) had no c-kit expression. Most of the c-kit immunoreactivity was varied 
and weak. Mast cells stained positive in all tissues (15/15) and served as an internal positive control (Figure 2a-f). The salivary gland that was present in two OL connective tissue stroma $(50 \%)$ showed weak focal staining (Figure $2 \mathrm{e}$ ) and the other $50 \%$ was negative (Figure $2 \mathrm{f}$ ).

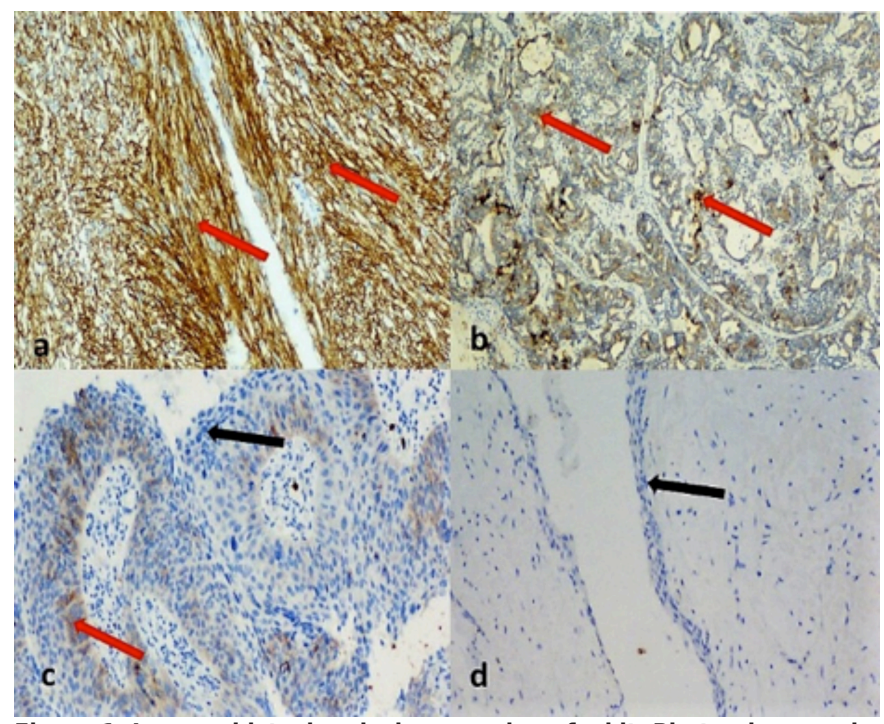

Figure 1: Immunohistochemical expression of c-kit. Photomicrographs showing strong expression with GIST (a), Cytoplasmic expression in ACC solid form (b), Cytoplasmic c-kit expression for SCC of cervix (c), no expression with DC (d).

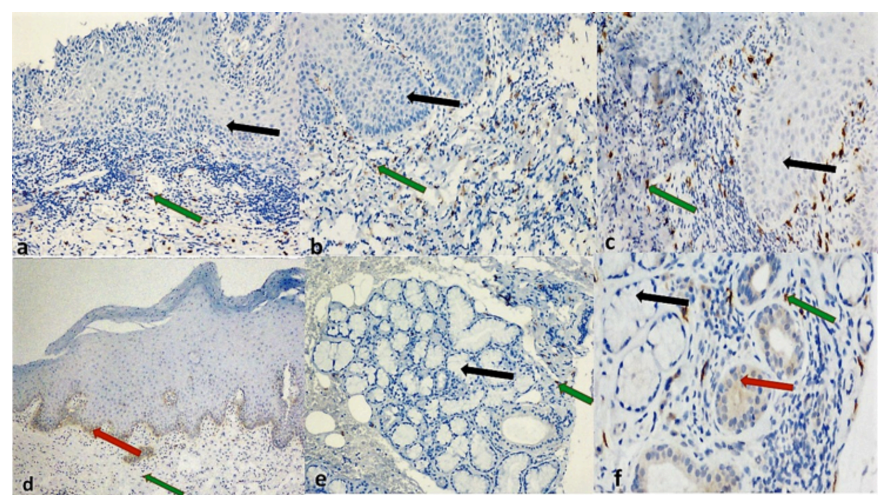

Figure 2: Immunohistochemical expression of c-kit. Photomicrographs showing no expression in OSCC (a), no expression with OED (b), Mast cells showing strong intensity and servedasan internal positive control no expression with OED (c), cytoplasmic expression in basal cells with the weak intensity of staining (d), no expression withsalivaryglands(e) weakcytoplasmicexpressioninsalivaryglands(f). (*Red arrow-expression of c-kit, blackarrow-no expression, green expression of ckitformastcells).

Histological grading of OED is the gold standard for malignant transformation, but it iscontroversial due to interobservervariability. ${ }^{1}$ The relationship between the type of epithelial dysplasia and cancer progression is yet to be known. ${ }^{1}$ The KIT proto-oncogene receptor tyrosine kinase is also known as c-kit, kit, or stem cell factor. The receptor is a 145- to 165 -Kd proto-oncogene that is structurally linked to the colony-stimulating factor 1 and platelet-derived growth factor receptors. This is required for normal haematopoiesis, melanogenesis, and gametogenesis. As a result, the c-kit gene product is expressed in many normal cell types, including mast cells. ${ }^{2}$ CD117 is a hematopoietic stem and progenitor cell marker, whose expression has been reported in a variety of cancers, including mesenchymal, epithelial, and haematological cancers. ${ }^{2,3}$

Two general pathways of CD117 activation have been described for cancers: Acquiring activating mutations that result in constitutive ligand-independent phosphorylation and a possible autocrine and/or paracrine stimulation of the receptor by its ligand, stem cell factor, has been proposed..$^{2-4}$ It is yet unknown how CD117 activation influences proliferation, stemness, and migration. There are some variances in detectable c-kit expression. However, c-kit expression is lower in the majority of carcinoma-like pleomorphic carcinomas $(0.6 \%)$, SCC $(3.4 \%)$, breast cancer $(21 \%)$, hepatocellular carcinomas $(17 \%)$, large cell carcinomas $(5.5 \%)$, while ovarian tumours (81\%) and adenoid cystic carcinoma $(100 \%)^{4,5}$ indicating that secretory organs have higher levels of c-kit expression. C-kit is widely expressed in head \& neck SCC; but not in normal mucosa, a substantially higher expression in pharyngeal SCC than in laryngeal SCC. The biological relevance of this finding is uncertain, but it could be related to the laryngeal and pharyngeal embryologic derivatives. Normal squamous epithelium of the pharynx and larynx does not express. ${ }^{4}$

In a recent study, it was observed that the positive expression of the c-kit was reduced in the OSCC $(5 / 84,5.9 \%)$ and in the OED $(1 / 9,11 \%)$ group, completely negative in the control group of normal oral mucosa $(5 / 5,100 \%) .{ }^{5}$ In other research, no CD117 reactivity was observed in either normal oral tissue or carcinomatous proliferations; whereas, reactivity restricted to stromal cells. ${ }^{3}$ Only $19.4 \%$ of SCCs (6/31) expressed focally, and expression was limited to the $13 \%$ tumour's basal layer (4/31); whereas, all porocarcinomas (22/22) were positive. ${ }^{6}$ Immunoreactivity was detected in $71 \%$ of adenoid cystic carcinomas (25/35). One hundred percent negative expression (5/5) in normal salivary gland tissues demonstrates the significant, uncommon c-kit expression in solid tumours; whereas, secretory organs have greater c-kit expression. ${ }^{2}$ It is apparent that CD117 is not a reliable marker for identifying a population of cancer stem cells in solid tumours, since it does not characterise tumour-initiating cells, and appears to be more restricted in identifying mesenchymal stem cells. ${ }^{3}$ Although there are few and contradictory data on CD117 reactivity in squamous cell carcinoma, CD117 expression was not observed in normal squamous epithelium of the pharynx, larynx, oral tissue, or salivary gland, according to the literature..$^{2-4}$ In the current research, OSCC and the epithelium of DC did not express c-kit expression. Expression was observed only in 24.6\% (04/15) of cases of oral leukoplakia, predominantly in the basal layer, and the immunoreactivity was varied and weak. Despite the presence of mild localised staining of oral leukoplakia epithelial cells, the immunoreactivity was lower than the cut-off value to consider them as positive. All samples of OL $(n=15 / 15)$ expressed positive for stromal cells, with strong intensity of staining. The current study's possible drawback was that it was conducted retrospectively and dealt with archival histopathology specimens. 
Although four cases of OL revealed weak expression of c-kit, this may not be a specific marker to distinguish between oral leukoplakia with a high risk for malignant transformation. More research is needed to determine whether c-kit expression in some solid tumours and oral leukoplakia is caused by c-kit gene mutations or is an epigenetic phenomenon, that warrants further investigation to increase the sensitivities and specificities for the detection of CSCs in OL. Prospective studies on a larger number of cases are recommended.

\section{FUNDING:}

This study was financially supported by a SEGi University Research Grant (SEGiuni/VC/RIMC/33/19-12-2016).

\section{CONFLICT OF INTEREST:}

The authors declared no conflict of interest.

\section{AUTHORS' CONTRIBUTION:}

AST: Design of the work, analyses and interpretation of data for the work.

VHS: Drafting the work or revising it critically for important intellectual content and manuscript editing.

SMBI, MTA: Data collection and final approval of the version to be published.

BAF: Manuscript editing.

ATBZ: Approval for research work.

\section{REFERENCES}

1. Speight PM, Khurram SA, Kujan O. Oral potentially malignant disorders: Risk of progression to malignancy. Oral Surg Oral Med Oral Pathol Oral Radiol 2018; 125(6):612-27. doi: 10.1016/j.0000.2017.12.011.

2. Ahmed MM, Abo-Hager EA. Differential expression of c-kit and CD43 in histological subtypes of adenoid cystic carcinoma of salivary gland. Saudi Dent J 2010; 22(1):27-34. doi: 10.1016/j.sdentj.2009.12.001.

3. Mărgăritescu C, Pirici D, Simionescu C, Stepan A. The utility of CD44, CD117 and CD133 in identification of cancer stem cells (CSC) in oral squamous cell carcinomas (OSCC). Rom J Morphol Embryol 2011; 52(3):985-93.

4. Ongkeko WM, Altuna X, Weisman RA, Wang-Rodriguez J. Expression of protein tyrosine kinases in head and neck squamous cell carcinomas. Am J Clin Pathol 2005; 124(1):71-6. doi: 10.1309/BTLN5WTMJ3PCNRRC.

5. Sathiyamoorthy J, Shyamsundar V, Shanmugam S, Mani J, Hari R. Immunohistochemical expression of c-kit in oral squamous cell carcinoma patients in south Indian populations. Asian J Pharm Clin Res 2018; 11(10): 247-50.

6. Goto K, Takai T, Fukumoto T, Anan T, Kimura T, Ansai S, et al. CD117 (KIT) is a useful immunohistochemical marker for differentiating porocarcinoma from squamous cell carcinoma. J Cutan Pathol 2016; 43(3):219-26. doi: 10.1111/cup.12632. 